\title{
Research on Development Path of Culinary Specialty in Secondary Vocational Schools Based on Data Mining Technology COVID-19 Epidemic Background
}

\author{
Yunjie Zhao ${ }^{1, *}$ \\ ${ }^{1}$ Institute of Educational Economics and Management, Tongji University, Shanghai, Jiading District 201805, China \\ *Corresponding author.Email: zyj9863@163.com
}

\begin{abstract}
The 2020 COVID-19 epidemic has significantly affected China's domestic education field, including secondary vocational education. At the same time, in secondary vocational schools, the culinary major is both traditional and long-term developmental. In the context of the COVID-19 epidemic, the conduct and development of professional teaching activities are facing certain difficulties. With the help of data mining technology, effective analysis of the culinary profession in the context of the COVID-19 epidemic has become a key proposition for how to better build a culinary profession under the COVID-19 epidemic. Keywords: data mining, The outbreak, China, Vocational education, Professional cooking
\end{abstract}

\section{INTRODUCTION}

\subsection{Outbreak and Spread of COVID-19}

The sudden COVID-19 epidemic is a major public health emergency with the fastest spread, the widest range of infections, and the most difficult prevention and control since the founding of the People's Republic of China. The COVID-19 epidemic posed a severe challenge to China's vocational education industry.

On the one hand, the COVID-19 epidemic is sudden. In a relatively short period of time, the sudden outbreak of the COVID-19 epidemic has made it necessary for secondary vocational schools to change their teaching methods in a timely manner during the overall development process. This change has implications for courses, teachers, students, and teaching environments. Teaching methods, study habits, etc. all have an impact. On the other hand, the spread of the COVID-19 epidemic is widespread, which is not only reflected in the length of time, but also in the spread and radiation of space, which has caused the quality of secondary vocational schools to be hindered.1.2The particularity of culinary majors.

\subsection{The Particularity of the Culinary Profession}

As a "sunrise" profession that is both traditional and sustainable, the cooking profession aims at cultivating chefs, executive chefs, and senior culinary talents. It is good at management, comprehensive technology, and professional cooking with good comprehensive qualities.
Talent is the goal. Therefore, compared with other majors, such as accounting, management, etc., cooking majors pay more attention to cultivating students' hands-on skills. A survey of 95 secondary vocational schools offering cooking majors in Shanghai, Zhejiang, and Fujian provinces found that $83.89 \%$ of them the courses are all conducted in the training room, and the proportion of the time for theoretical knowledge learning is relatively low. However, the new crown pneumonia epidemic has caused difficulties in the development of offline training and teaching.

\subsection{Extensive Application of Data Mining Technology}

Data mining is a cross-discipline formed by the integration of statistics, artificial intelligence, database, visualization technology and other fields. At present, it has been widely used in various fields, and the object of data mining can be any type of data source. It can be a relational database, such a data source containing structured data; it can also be a data warehouse, text, multimedia data, spatial data, time series data, Web data, such a data source containing semi-structured data or even heterogeneous data.

\section{DATA MINING TECHNOLOGY IS USED TO ANALYZE THE SITUATION OF COOKING SPECIALTY IN VOCATIONAL EDUCATION UNDER THE COVID-19 EPIDEMIC SITUATION}

Data mining refers to the process of searching for information hidden in a large amount of data through algorithms. It contains more of them. In view of the 
special background of the new crown pneumonia epidemic, select individual methods to conduct in-depth investigations on 95 secondary vocational schools that offer cooking majors in Shanghai, Zhejiang, and Fujian provinces, and based on the research. Above, use appropriate data mining techniques for analysis.

\subsection{Snow NLP Sentiment Analysis}

In the period from January 2020 to February 2021, 569 teachers and students of 195 professional students majoring in cooking in Shanghai, Zhejiang, and Fujian provinces were surveyed and interviewed. Through interviews, the interview questions were mainly designed as His subjective reaction of teachers and students in teaching under the new crown pneumonia epidemic. Using Snow NLP's emotional tendency analysis method in data mining technology, the emotional tendency contained in the subjective interview text is extracted and classified into two types: positive and negative. Use sentiments () function to perform sentiment analysis on text. The result returned by the function is a floating-point number between $0-1$. The closer to 1 the more positive (forward), and the closer to 0 the more negative (reverse). As shown below.

\section{Analysis of teachers' emotional tendenvy}

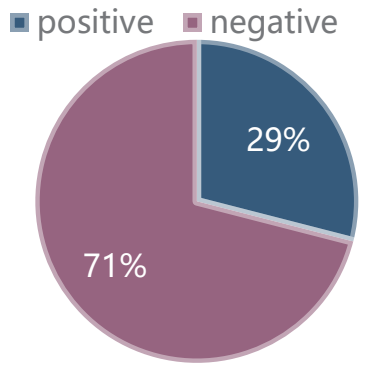

Figure 1. Analysis diagram of emotional tendency of cooking teachers of vocational students

\section{Analysis of students' emotional tendenvy}

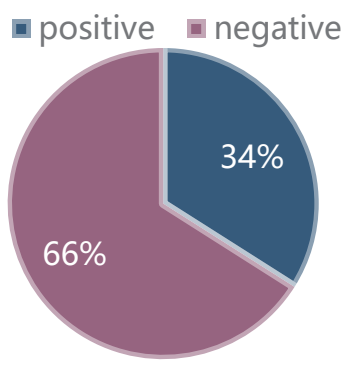

Figure 2. Analysis diagram of emotional tendency of cooking majors of vocational students

\subsection{Word Frequency Statistics}

In response to the new crown pneumonia epidemic, multiple subjects participating in the vocational education culinary profession, including students, teachers, parents, and cooking-related companies, a total of 296 personnel collected interview content, and used Python Chinese word segmentation component-"jieba" Chinese word segmentation to perform data Word segmentation operation. For the obtained data segmentation results, read each segmentation in the program loop. Define a thesaurus in the form of a dictionary. After counting all the word segments, calculate the proportion of each type of word segmentation, that is, count the word frequency. And take out the top ten segmentation words with the largest proportion as shown in the figure below.

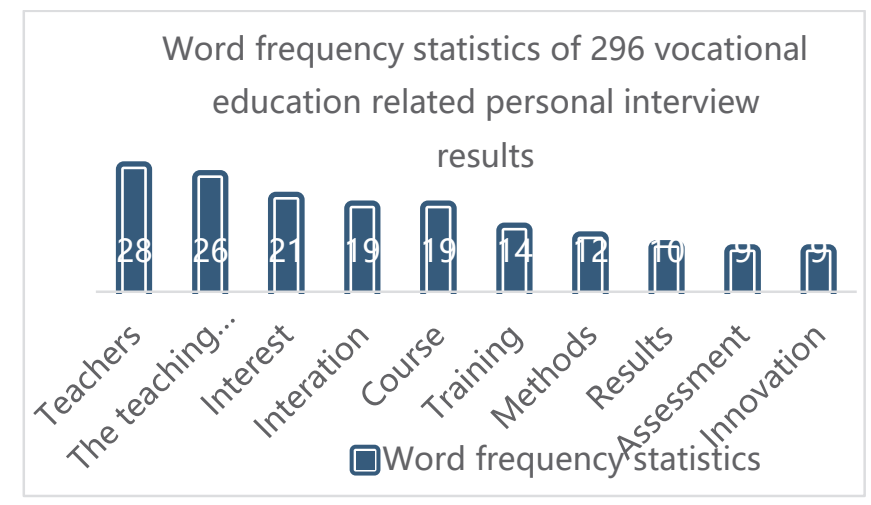

Figure 3. Statistical chart of word frequency of 296 professional cooking professionals interviewed

Thus, different main body under the outbreak, for the development of the vocational school professional cooking, the most attention is the "teacher" "teaching" and "interest" and other factors, need in the later model and system developed, focus on this kind of factor, to better meet the demand, thus boosting vocational education professional cooking whole conduct under the outbreak.

\section{FORMULATE A UNIQUE CULINARY SPECIALTY CONSTRUCTION MODEL SUITABLE FOR THE COVID-19 EPIDEMIC PERIOD}

\subsection{Policies and Documents to Support the Culinary Profession}

Due to the lack of clear directions and goals in the overall development of the cooking profession under the new crown pneumonia epidemic, it is necessary to increase assistance and support in policies and documents. First of all, the national macro level needs to formulate documents for secondary vocational schools against the background of the new crown pneumonia epidemic. At the same time, 
in addition to formulating corresponding policies and regulations at the national level, it is also necessary to increase efforts at the level of policy implementation. Secondly, due to the impact of the new crown pneumonia epidemic, various types of industrial production have been significantly affected. Therefore, the regional government also needs to base on the background of the new crown pneumonia epidemic to measure and measure the types of industries and industrial layout in the region. analyze. Finally, at the secondary vocational school level, it is also necessary to conduct in-depth research on the multiple subjects in the school, including students, parents, and teachers

\subsubsection{Increase the Investment in Capital}

First of all, because the daily teaching of cooking majors involves more professional cooking equipment, including "steaming cabinets", "clay pots", "water tables" and "woks", etc. However, due to the long duration of the new crown pneumonia epidemic, , The related equipment needs certain maintenance and care. Secondly, related funds need to use online teaching equipment and technical investment. Under the new crown pneumonia epidemic, online teaching needs to invest more technical financial assistance, such as "3D cooking kitchenware" and "VR virtual training room". and many more. Finally, related funds are also used in the development of courses. It is necessary to purchase brand-new equipment and equip with updated teaching materials. The use of related funds includes equipping students with teaching equipment, development and binding of teaching materials, and so on.

\subsubsection{Change of course content}

Under the new crown pneumonia epidemic, it is necessary to design and develop new curriculum content and add different types of curriculum formats. In terms of course content, it is necessary to delete courses that are less effective in improving students' comprehensive level and develop students' multiple intelligences, and add specific courses under the background of the new crown pneumonia epidemic, such as "SWOT self-assessment courses" and "career planning courses". On the other hand, in the form of course development, it is also necessary to innovate, such as offering "online virtual training courses" and so on

\subsubsection{Adjustment of faculty allocation}

Therefore, it is necessary to form a team of professional cooking teachers who can adapt to the background of the new crown pneumonia epidemic and have comprehensive capabilities. This requires secondary vocational schools to adjust teachers' recruitment conditions, access mechanisms, training content, and assessment methods.
For example, giving priority to teachers with better computer skills in the recruitment process can provide support for online teaching. At the same time, in the process of teacher training, attention should be paid to various abilities, such as "employment guidance ability" and "psychological adjustment." "Ability", "Emergency Capability", "Computer Operation Capability" and so on.

\subsection{Consider the Emotional Changes of Multiple Subjects}

Therefore, different subjects have different reasons for emotional fluctuations, and they need to be emotionally adjusted in the overall teaching activities according to different subjects. For example, you can design a new set of Snow NLP emotional tendency test software, configure online teaching courses, different subjects according to their own identity, test the current self-emotional tendency, and then the system will match their emotional tendency according to the relevant test. And make specific suggestions. For example, make suggestions on the learning environment, learning time, learning methods, etc.

\subsection{Carry Out School-Enterprise Cooperation Under Special Background}

The new crown pneumonia epidemic has significantly affected the overall development of my country's industry. As a relatively special type of industry, the catering industry is facing greater difficulties under the obstacle of the new crown pneumonia epidemic. However, under this special background, the cooking major of secondary vocational schools and the related catering industry cannot interrupt related school-enterprise cooperation activities but must adjust the form and continue to promote the in-depth integration of industry and secondary vocational schools. For example, both schools and enterprises can also discuss scientific research together in the form of online meetings and develop new software, courses, and equipment, which are not only suitable for the new crown pneumonia epidemic environment, but also provide ideas for the future integration of industry and education.

\section{CONCLUSION}

In summary, under the new and special background of the new crown pneumonia epidemic, it is extremely necessary to conduct a more accurate assessment of the development of the cooking major of secondary vocational schools. At the same time, the use of data mining technology to conduct an in-depth analysis of the current situation and difficulties faced by the culinary profession under the new crown pneumonia epidemic is reasonable and feasible. In the future, when developing the culinary profession in the context of the epidemic, it is necessary to do a good job in 
supporting the culinary profession in policies and documents, increase financial investment, improve the original teaching model, consider the emotional changes of multiple subjects, and develop schools under special backgrounds. Enterprise cooperation can better lead the development of secondary vocational education.

\section{REFERENCES}

[1] Cai Shunlin. Practical Exploration of Modern Apprenticeship Talent Training Mode for Secondary Vocational Cooking Major [J]. Vocational and Technical Education, 2018, 39(29):29-32.

[2] Ma Laijun. On-site Embedded Engineering Cycle-A New Approach to Integrating Engineering with Medical Professionals [J]. Vocational Education Forum, 2008(08):10-15.

[3] Shen Jianping. Practice and Research on the "Enterprise" Operation Management of Campus Characteristic Culture in Vocational Schools-Case of
School-Enterprise Culture Docking in Hangzhou Cooking Major of Secondary Vocational Schools [J]. China Vocational and Technical Education, 2011(25):30-32+35.

[4] Duan Feng, Zheng Xiaoqi, Zhou Wenyong. Hangzhou Zhongce Vocational School Culinary Specialty Project Curriculum Development [J]. Vocational and Technical Education, 2008, 29(05):5+94.

[5] Cheng Wei. Exploration on Modern Apprentice Training of Cooking Specialty Based on Innovative Craftsman "Customization" [J]. Food Industry, 2017, 38(12):223-226.

[6] LIU Xiao, SHAO Wenqi. Investigation on the Status Quo of Vocational College Internship Promoting Enterprise Restoration under the Background of Anti-COVID-19 epidemic [J]. Analysis from the Perspective of 319 Secondary Vocational Teachers. Education and Occupation, 2020(14):33-39. 